\title{
PROPUESTA DE INTERVENCIÓN PSICOSOCIAL EN AUTODETERMINACIÓN, HABILIDADES SOCIALES Y CAPACIDAD EMPRENDEDORA: CONTRIBUCIÓN DESDE LA PSICOLOGÍA A LA INTEGRACIÓN DE MUJERES MICROEMPRESARIAS CON DISCAPACIDAD INTELECTUAL
}

\author{
PSYCHOSOCIAL INTERVENTION PROPOSAL IN SELF-DETERMINATION, SOCIAL SKILLS AND \\ ENTREPRENEURIAL CAPACITY: CONTRIBUTION FROM PSYCHOLOGY TO THE INTEGRATION OF \\ ENTREPRENEURS WOMEN WITH INTELLECTUAL DISABILITIES
}

Yohanna del Pilar Poblete TOlOZA*, ANDRÉS EDUARdo JimÉNEZ FigUEROA**

UniversidAd DE TALCA, TALCA - Chile

FECHA RECEPCIÓN: 17/7/2013・ FECHA ACEPTACIÓN: 27/8/2013

Para citar este artículo: Poblete, Y. \& Jiménez, A. (2013). Propuesta de intervención psicosocial en autodeterminación, habilidades sociales y capacidad emprendedora: contribución desde la psicología a la integración de mujeres microempresarias con discapacidad intelectual. Psychologia: avances de la disciplina, 7(2), 55-67.

\begin{abstract}
Resumen
Se elabora una propuesta de intervención en bienestar subjetivo, autodeterminación, habilidades sociales y capacidad emprendedora en mujeres microempresarias con discapacidad intelectual a partir de un diagnóstico realizado en 20 mujeres con discapacidad intelectual. Se administró una batería de cinco instrumentos compuesta por la escala de satisfacción con la vida de Diener et ál. (1984), escala de felicidad subjetiva de Lyubomirsky y Lepper (1999), escala de autoconcepto de la Rosa y Díaz (1999), cuestionario de habilidades sociales de Goldstein (1999) y cuestionario de emprendimiento de Spencer y Spencer (1993). Los principales resultados descriptivos indican que las mujeres de la muestra manifiestan altos niveles de desarrollo de su autoconcepto (autodeterminación) $(x=382,6 ; \sigma=53,32)$, habilidades sociales $(x=$ $91,6 ; \sigma=6,65)$ y bienestar subjetivo, tanto en felicidad subjetiva $(x=21,45 ; \sigma=3,96)$ como en satisfacción vital $(x=27,85 ; \sigma=5,79)$ y niveles moderados de su capacidad emprendedora $(x=65,85 ; \sigma=5,66)$. Se obtuvo un adecuado nivel en todas las variables en las mujeres de la muestra, enfocando la propuesta de intervención en potenciar sus habilidades y competencias, desarrollando conocimientos paralelos que apoyen a los ya existentes.

Palabras clave. Discapacidad intelectual, bienestar subjetivo, autodeterminación, habilidades sociales, emprendimiento.
\end{abstract}

* MA en Psicología Social y Psicóloga Organizacional. Facultad de Psicología, Universidad de Talca. E-Mail: ypoblete@utalca.cl

** MA en Administración y Dirección de Recursos Humanos y Psicólogo Organizacional. Docente de la Facultad de Psicología de la Universidad de Talca.

E-Mail: anjimenez@utalca.cl. Facultad de Psicología de la Universidad de Talca. Avda. Lircay s/n, Talca, Chile 


\begin{abstract}
It constructs an intervention on subjective well-being, self-determination, social skills and entrepreneurship in women microentrepreneurs with intellectual disabilities from a diagnosis realized in 20 women with intellectual disabilities.It administered a battery of five instruments: Satisfaction Scale life Diener et ál (1984), subjective happiness scale Lyubomirsky and Lepper (1999), Self-Concept Scale (La Rosa y Díaz, 1999), Questionnaire social skills Goldstein (1999) and Entrepreurship Questionnaire (Spencer \& Spencer, 1993). The descriptive analysis indicates that women in the sample expressed high levels of development of their self-concept (self-determination) ( $\mathrm{x}=382.6$, $\sigma=53.32)$, social skills $(\mathrm{x}=91.6, \sigma=6,65)$ and subjective well-being, both in subjective happiness $(\mathrm{x}=21.45, \sigma=3.96)$ and life satisfaction $(x=27.85, \sigma=5.79)$ and moderate levels of entrepreneurial ability $(x=65.85, \sigma=5,66)$. An adequate level for all variables in women in the sample, the proposed intervention focusing on enhancing their skills and competencies, developing parallel knowledge to support existing ones.

Keywords. Intellectual disability, subjective well-being, self-determination, social skills, entrepreneurship.
\end{abstract}

\section{Introducción}

Las personas que presentan condiciones especiales han sido víctimas de discriminación y de no inclusión social desde que se tiene conciencia y registros (Cordeu, 2008).

En Chile, más de 2000000 de personas padecen una discapacidad en cualesquiera de sus grados, donde uno de cada tres hogares tiene un miembro con tales características (FONADIS, 2006).

Por más de dos décadas ha surgido la necesidad de adoptar prácticas efectivas para entregar seguridad a las personas (Detrich, Keyworth \& States, 2008), lo que se ha extrapolado a individuos con discapacidad, demostrándose que la autodeterminación, orientada a derechos, competencias, acciones, ética, responsabilidad, dignidad y libertad de estos individuos (Tamarit, 2001), mejora al difundir herramientas que generen conductas y comportamientos más efectivos en ellos (Bacon, Walker, Schwartz, O`Hara, Calkins \& Wehmeyer, 2011).

De esta manera, cuando las personas con discapacidad logran mantener adecuados niveles de autodeterminación, es necesario indagar en el bienestar subjetivo que presentan, puesto que permite conocer las respuestas emocionales y juicios globales que tienen frente a la vida (Diener, Suh, Lucas \& Smith, 1999). Junto con ello, son de suma relevancia las habilidades sociales, las que pueden ser entrenadas mediante la interacción de factores intrínsecos de la persona y factores ambientales (Boluarte, Méndez \& Martell, 2006).

Ahora bien, para fines de este diagnóstico se ha considerado como grupo objetivo a mujeres microempresarias con discapacidad intelectual para medir su capacidad emprendedora, ya que según la teoría, es el género femenino quien emprende en mayor medida en relación al género masculino (De la Red, Fernández, Marbán \& de la Puente, 2008). Asimismo, resulta interesante entrenar sus habilidades sociales, pues fortalecen su autoestima, autodeterminación y se adaptan con mayor facilidad a contextos de integración social y laboral (Boluarte et ál., 2006).

Por tanto, el propósito de la propuesta de intervención será entregar herramientas relacionadas con autodeterminación, habilidades sociales y emprendimiento en mujeres microempresarias con discapacidad intelectual, dejando obsoletos los estigmas que aminoran su nivel de satisfacción y de felicidad (Boluarte et ál., 2006), logrando así mejores niveles de bienestar subjetivo y disminuyendo la escasa conciencia social que existe en las organizaciones cuando deben cubrir las necesidades de las personas con discapacidad (Ararteko, 2003).

\section{Relevancia de las variables discapacidad, auto- determinación, habilidades sociales y capacidad emprendedora sobre el bienestar subjetivo}

La discapacidad es vista como una deficiencia o minusvalía construida a partir de imágenes mentales que tienen relación con limitaciones y carencia de oportunidades de desarrollo humano (Zondek, Zepeda, González \& Recabarren, 2006). De esta manera, alcanzar la integración laboral de las personas con discapacidad es una de las iniciativas prioritarias hoy en día, buscando la igualdad de oportunidades y derechos, satisfacer las demandas y seguir acumulando experiencias positivas en el desenvolvimiento laboral normalizado de estos individuos (Verdugo, Jordán, Vicent \& Martin, 2006). Es por ello que las personas con esta condición han sido claves en las políticas sociales establecidas en la cultura 
occidental, que en la actualidad se basan en cuatro puntos fundamentales, que son: igualdad de oportunidades, participación total, vida independiente y autosuficiencia económica.

Ahora bien, es de suma relevancia verificar el autoconcepto que presentan las personas con discapacidad, puesto que para intervenir en ellas es necesario conocer los recursos y herramientas que tienen previamente, sobre todo a nivel de autoestima. Es por ello que la autodeterminación ha sido definida por Wehmeyer (1996) como el principal agente causal de la vida de un individuo y hacer elecciones y tomar decisiones respecto a la calidad de vida propia, sin influencias o interferencias externas innecesarias. Además, las acciones autodeterminadas que realizan las personas tienen a la base cuatro características, que son: autonomía, autorregulación, fortalecimiento/capacitación psicológica (empowerment) y autorrealización (Wehmeyer, 1996, 2001; Wehmeyer, Kelchner \& Richards, 1996).

La autodeterminación, entonces, se ha utilizado para guiar las prácticas en programas y servicios destinados a personas con discapacidad, brindándoles bienestar social, educación especial y servicios de apoyo (Verdugo, n. d.), debido a que es un concepto factible de ser enseñado al dirigir y fomentar el aprendizaje de habilidades de autodeterminación, sobre todo en contextos escolares (Doll, Sands, Wehmeyer \& Palmer, 1996; Palomo \& Tamarit, 2000). La importancia que se le atribuye a la conducta adaptativa como predictor de la calidad de vida, al permitir que una persona con discapacidad intelectual adquiera las destrezas necesarias que promueven una conducta más independiente, el de autodeterminación (Stancliffe \& Abery, 2003), lo que implica incluir en los programas de apoyo al menos uno de los principios de calidad de vida en personas con discapacidad intelectual (Montero \& Lagos, 2011).

Asimismo, desarrollar la capacidad de interactuar con otros es muy importante para reforzar el autoconcepto de las personas con discapacidad. De esta manera, Caballo (1999) menciona que se deben desarrollar las habilidades sociales centrándose en las conductas que emite el individuo, en un contexto interpersonal, las que permiten expresar sus sentimientos, actitudes, deseos, opiniones o sus propios derechos, logrando la adecuación a la situación, respetando esas conductas en los de- más, resolviendo los problemas inmediatos del contexto y minimizando la probabilidad de dificultades futuras.

Ahora bien, el entrenamiento en habilidades sociales se ha utilizado en varias oportunidades en personas con discapacidad intelectual, logrando: 1) favorecer una integración y desinstitucionalización exitosa, 2) evitar la aparición de problemas de comportamiento, 3) prevenir la aparición de problemas psicológicos, 4) prevenir el abuso sexual (Gutiérrez \& Prieto, 2002). De esta manera, el poder entrenar habilidades sociales en las mujeres con discapacidad del estudio, permitirá, según lo planteado por Hinojo y Fernández (2002) que logren relacionarse con otros de manera efectiva, determinando en gran medida la búsqueda de su felicidad personal y bienestar subjetivo, lo que se consigue solamente mediante experiencias que modelan las conductas de estas personas.

Cabe destacar que, al tener un óptimo desarrollo de la autodeterminación y de las habilidades sociales, las mujeres con discapacidad demostrarían tener mayores capacidades para el desarrollo de su propio negocio. Es así que el emprendimiento es definido como un proceso social que surgió hace más de tres décadas y que se ha convertido en la fuerza económica de mayor potencial en el ámbito laboral (Verheul, Wennekers, Audretsch \& Thurik, 2001), transformándose en un concepto integrador entre las estrategias individuales y la innovación grupal, generando una perspectiva que ha revolucionado la forma de hacer negocio (Kuratko, 2005). Así, una emprendedora debe presentar: autoestima y autoconfianza, iniciativa, capacidad resolutiva, toma de decisiones, capacidad de asumir riesgos, de comunicación y negociación, de liderazgo y de trabajo en equipo, espíritu de autosuperación, resistencia a la presión y perseverancia (De la Red, Fernández, Marbán \& de la Puente, 2008).

De esta forma, la relación que las variables antes mencionadas tienen con el bienestar subjetivo radica en que las mujeres se sentirían más conformes con su forma de vida, considerando la definición que Cuadra y Florenzano (2003) hacen de dicho bienestar, como lo que las personas piensan y sienten con respecto a sus vidas y a las conclusiones, tanto cognitivas como afectivas, que alcanzan cuando evalúan su existencia, manifestando dos componentes principales: aspectos afectivo-emocionales y aspectos cognitivo-valorativos, encontrándose relacionados entre sí (Diener, 1994). 
Por una parte, la felicidad subjetiva, que es el componente afectivo del bienestar subjetivo, se refiere a todas aquellas emociones positivas o sentimientos de satisfacción y, a su vez, las posibilidades que las personas tienen para acceder a una vida favorable y positiva (Marinić \& Brkljačić, 2008), y por otra, la satisfacción vital, que es el componente cognitivo del bienestar subjetivo y se refiere a la valoración positiva que la persona tiene de su propia vida, ya sea a nivel general como específico (Diener, 1994; García, 2002; Diener, Suh, Lucas \& Smith, 1999; García-Viniegras \& González, 2000; Liberalesso, 2002), siendo los elementos de funcionamiento más cercanos a la vida personal del individuo los que influyen en mayor medida sobre su bienestar (Moyano-Díaz \& Ramos, 2007).

Existen fundamentos teóricos que dan cuenta de la relevancia de generar programas de intervención destinados a personas con discapacidad, es así que por más de dos décadas ha surgido a nivel social la necesidad de llevar a cabo prácticas efectivas que permitan entregar a estas personas seguridad (Detrich, Keyworth \& States, 2008) y, por ende, capacidad para mejorar sus estilos de vida (Tamarit, 2001). Ahora bien, la elección de trabajar con mujeres con discapacidad se justifica porque es el género femenino el que emprende en mayor medida en relación al género masculino (De la Red, Fernández, Marbán \& de la Puente, 2008).

De esta manera, lograr una óptima entrega de herramientas en autodeterminación, habilidades sociales y capacidad emprendedora en las mujeres del estudio, permitirá su crecimiento personal y profesional, al mismo tiempo que un potenciamiento social y laboral respecto de la inclusión que se puede generar con ellas. Es así que existe evidencia de trabajos destinados a personas con algún tipo de discapacidad que respaldan este estudio, como el de Ledo (2011), quien ha diseñado un proyecto dirigido a mujeres con discapacidad intelectual cuyo objetivo es fomentar la participación de estas a través de la elección de ocupaciones significativas, favoreciendo con ello su salud y bienestar, y fomentando su autonomía personal en el entorno social en el cual se desenvuelven.

\section{Modelo operativo de calidad de vida}

El modelo de calidad de vida de Schalock y Verdugo (2002/2003) propone un conjunto de dimensiones reconocidas como valores esenciales en todas las culturas (Junta de Castilla y León, 2004) a través de tres niveles sociales: microsistema (contexto social inmediato de la persona, principalmente vínculos familiares), mesosistema (vínculos con la comunidad e instituciones en un contexto más amplio que el familiar) y macrosistema (valores y creencias compartidos por la sociedad en torno a lo cultural, social, político y económico) (Peñas, Torres \& Gómez, 2005). A continuación la figura 1 muestra los niveles del modelo de calidad de vida.

De esta manera, el modelo operativo de calidad de vida busca impulsar la aplicación del concepto en programas y servicios para mejorar la atención centrada en las personas con discapacidad, contando con ocho dimensiones: autodeterminación (metas y valores personales), bienestar emocional (felicidad y satisfacción), bienestar físico (salud y cuidados básicos), bienestar material (tener y disfrutar de pertenencias), relaciones interpersonales significativas (amigos y familia), inclusión social (participar en la comunidad y apoyos sociales), desarrollo personal (desarrollar habilidades y competencias) y derechos (libertades y derechos legales y humanos) (Schalock \& Verdugo, 2002/2003).

En el contexto estudiado, mediante el uso de este modelo en la propuesta de intervención psicosocial destinada a las mujeres con discapacidad intelectual, se lograría dar cobertura a los tres ámbitos principales, además de generar una potenciación de las dimensiones que se plantean con el fin de mejorar su calidad de vida y, por sobre todo, su inclusión social-laboral, dado que son ellas las que deben adaptarse a las condiciones que les ofrece el mercado para ser valoradas por su trabajo, desempeño, habilidades y competencias, eliminando así las barreras sociales que existen para dar oportunidades de crecimiento frente a una condición de discapacidad.

\section{Objetivos de diagnóstico}

El objetivo general pretende realizar un diagnóstico y una propuesta de intervención del bienestar subjetivo, autodeterminación, habilidades sociales y capacidad emprendedora en mujeres microempresarias que presentan discapacidad intelectual de la ciudad de Talca.

Los objetivos específicos son: describir los niveles de autodeterminación, de habilidades sociales, de emprendimiento y de felicidad subjetiva y satisfacción 
vital en las mujeres de la muestra, que permitan elaborar una propuesta de intervención psicosocial en dichas variables.

\section{Método}

\section{Muestra}

20 mujeres microempresarias que presentan discapacidad intelectual (nivel leve) de la ciudad de Talca, cuyas edades fluctúan entre 17 y 21 años (promedio de 18.05 años), todas pertenecientes a un Centro de Capacitación Laboral de la región. La muestra fue elegida por conveniencia, por la condición del estudio: ser mujer, presentar algún grado de discapacidad y ser microempresaria o tener conocimientos de emprendimiento.

\section{Tipo y diseño del diagnóstico}

Exploratorio descriptivo, con diseño metodológico no experimental y de corte transversal (Hernández, Fernández \& Baptista, 1998).

\section{Instrumentos}

Escala de Satisfacción con la Vida (Diener et ál, 1984). Está compuesta por un total de cinco ítems, que se responden a través de una escala tipo Likert, donde uno es muy en desacuerdo y siete, muy de acuerdo. El total del puntaje de la escala de satisfacción con la vida va de 5 a 35. Se utiliza la adaptación y validación de Moyano (2005) a muestras chilenas (927 personas), obteniendo una $\alpha$ de Cronbach de 0.87 y un coeficiente de Spearman Brown de 0.83, mientras que el $\alpha$ de Cronbach obtenido en el análisis de fiabilidad fue de 0.99 y un test retest después de 10 semanas de 0.83 , en estudiantes universitarios de pregrado.

Escala de Felicidad Subjetiva (Lyubomirsky \& Lepper, 1999). Está compuesta por cuatro ítems, que se responden de acuerdo a una escala de diferencial semántico de uno a siete, donde siete representa la mayor felicidad. Se utiliza la versión chilena con un $\alpha$ de Cronbach de 0.79 y de 0.77 en test-retest en una muestra de 133 participantes (Moyano-Díaz \& Ramos, 2007).

Escala de Autoconcepto (La Rosa \& Díaz, 1999). Está compuesta de 54 pares de adjetivos en los cuales uno es el antónimo del otro, referidos a cuatro dimensiones: social, emocional, ocupacional y ética. Cada par usa diferencial semántico con siete intervalos, precedidos por la expresión «Yo soy». La consistencia interna ( $\alpha$ de Cronbach) de las dimensiones es: ocupacional $(\alpha=0.80)$, ética $(\alpha=0.77)$, social $(\alpha=0.78)$ y emocional $(\alpha=0.71$ y 3 subdimensiones: estados de ánimo $<\alpha=0.86>$, sentimientos interindividuales $<\alpha=0.81>$ y salud ocupacional $\langle\alpha=0.76>$ ). Se opta por este instrumento, ya que incluye dimensiones que otras escalas no consideran y que resultan de gran relevancia para este estudio, específicamente el área social y ocupacional.

Cuestionario de habilidades sociales (Goldstein, 1999). Este cuestionario es el resultado de una serie de habilidades elaborado a partir de Goldstein y otros autores como Spencer, obteniéndose en Perú una confiabilidad de 0.916 (Lescano, Rojas \& Vara, 2003). Se utiliza la validación de González y Jiménez (2012) en una muestra chilena (170 mujeres), obteniéndose un $\alpha$ de Cronbach de 0,832 y una validez de constructo mediante análisis factorial con prueba KMO de 0,619 para los 37 ítems del cuestionario, donde los 13 primeros explican en un $64,6 \%$ el total de la varianza.

Cuestionario de emprendimiento (Spencer \& Spencer, 1993). Este cuestionario tiene siete dimensiones y un análisis de confiabilidad de 0,96. Se utiliza la validación de González y Jiménez (2012) en una muestra chilena (170 mujeres), obteniéndose un alfa de cronbach de 0,8 y una validez de constructo mediante análisis factorial con prueba KMO de 0,693 para los 29 ítems del cuestionario, donde los 10 primeros explican en un $63,8 \%$ el total de la varianza.

\section{Procedimientos del diagnóstico}

El procedimiento consta de cinco etapas, comenzado por la Sensibilización, donde se contactó a la directora de la organización y se consigue el permiso para realizar el diagnóstico. Posteriormente, se realiza el Contacto de las personas de la muestra, explicándoles al grupo de mujeres los objetivos del estudio y la confidencialidad de los datos, respondiendo a sus inquietudes. Luego, se realiza la Aplicación de la batería de instrumentos, reuniendo a las 20 mujeres de la muestra en una sala habilitada para ello con asistencia voluntaria, contestando la batería de instrumentos, teniendo el 
apoyo de una persona que ayudó a coordinar y revisar la forma de responder de estas. Durante la aplicación, una persona leyó cada una de las afirmaciones, explicando su significado y la forma en que debían responder. Se continúa con el Análisis de datos logrados en la aplicación, respondiendo así a los objetivos del estudio. Seguido de ello, se lleva a cabo la Elaboración de la propuesta de intervención psicosocial a partir de dichos datos en relación a las variables autodeterminación, habilidades sociales y capacidad emprendedora.

Cabe destacar que se realiza un análisis del Modelo operativo de calidad de vida planteado por Schalock y Verdugo (2002/2003) en relación a los resultados obtenidos en el diagnóstico, identificando las variables que se pueden potenciar y desarrollar en mayor medida del actual valor presentado, de acuerdo a sus tres niveles: microsistema, mesosistema y macrosistema (ver en detalle en apartado Conclusión y Discusión).

Por último, el presente estudio tiene en la base la idea de que todas las personas son iguales y, por ende, deben tener las mismas oportunidades en todo ámbito de su vida: social, familiar y laboral, velando porque sus derechos humanos sean respetados en cualquier situación o contexto. Es así que los requerimientos éticos se han considerado a partir de lo planteado por el Colegio de Psicólogos de Chile (2010), a saber esta investigación se adscribe a los siguientes artículos: Cap. 1, art. 1. : Respeto por los derechos y la dignidad de las personas. Se ha respetado la dignidad y los valores de todas las participantes, respetando sobre todo el derecho a la privacidad y confidencialidad de la información, Cap. 1, art. 6 ${ }^{\circ}$ : Responsabilidad social. Se considera lo relevante que es para la sociedad estudiar de forma responsable a las personas con discapacidad intelectual, con el fin de no dañar su integridad como individuos, Cap. 2, art.9. ${ }^{\circ}$ : Respeto por los otros. Se han respetado los valores, conductas, actitudes y opiniones de las participantes, Cap.3, art. 11. ${ }^{\circ}$, apartado 6. Confidencialidad. Se ha resguardado el uso de la información entregada por las participantes solo con fines investigativos del estudio.

\section{Resultados de diagnóstico}

En la tabla 1 se observa que las participantes presentan un nivel avanzado de su desarrollo personal, incluyendo la autoestima y autoconcepto $(x=382,6$; $\sigma=53,32)$.

Tabla 1. Descriptivos de la variable autodeterminación en las mujeres con discapacidad de la muestra.

\begin{tabular}{lccccccc}
\hline \hline & N & Mínimo & Máximo & Media & Error típico & Desviación estándar & Varianza \\
\hline Nivel de autoconcepto & 20 & 294 & 463 & 382,6 & 11,92 & 53,32 & 2842,67 \\
N válido & 20 & & & & & & \\
\hline \hline
\end{tabular}

En la tabla 2 se observa que las mujeres manifiestan un nivel avanzado en su capacidad para relacionarse con otros de manera efectiva y con adecuadas capacidades de desenvolvimiento interpersonal $(x=91,6 ; \sigma=6,65)$. Sin embargo, es posible apreciar en la tabla 3 diferencias en las dimensiones de las habilidades sociales, obte- niendo para la primera $(\mathrm{x}=14,90 ; \sigma=1,59)$, la segunda $(\mathrm{x}=14,85 ; \sigma=1,79)$ y la cuarta $(\mathrm{x}=13,85 ; \sigma=1,66)$ presentan un nivel medio de desarrollo, mientras que la tercera $(x=12,50 ; \sigma=1,32)$, la quinta $(x=15,55$; $\sigma=2,30)$ y la sexta $(x=19,95 ; \sigma=2,26)$ presentan un nivel medio casi avanzado de desarrollo.

Tabla 2. Descriptivos de la variable habilidades sociales en las mujeres con discapacidad de la muestra.

\begin{tabular}{lccccccc}
\hline \hline & N & Mínimo & Máximo & Media & Error típico & Desviación estándar & Varianza \\
\hline Nivel de HHSS & 20 & 78 & 101 & 91,6 & 1,49 & 6,65 & 44,25 \\
N válido & 20 & & & & & & \\
\hline \hline
\end{tabular}


Tabla 3. Descriptivos de las dimensiones de la variable habilidades sociales en las mujeres con discapacidad de la muestra.

\begin{tabular}{lccccccc}
\hline Dimensiones HHSS & N & Mínimo & Máximo & Media & $\begin{array}{c}\text { Error } \\
\text { típico }\end{array}$ & $\begin{array}{c}\text { Desviación } \\
\text { estándar }\end{array}$ & Varianza \\
\hline Primeras HHSS & 20 & 11 & 17 & 14,90 & 0,35 & 1,59 & 2,52 \\
HHSS avanzadas & 20 & 11 & 18 & 14,85 & 0,39 & 1,79 & 3,19 \\
HH relacionadas con los sentimientos & 20 & 10 & 14 & 12,50 & 0,29 & 1,32 & 1,74 \\
HH alternativas a la agresión & 20 & 10 & 17 & 13,85 & 0,37 & 1,66 & 2,77 \\
HH para hacer frente al estrés & 20 & 10 & 18 & 15,55 & 0,51 & 2,30 & 5,31 \\
HH de planificación & 20 & 15 & 23 & 19,95 & 0,50 & 2,26 & 5,10 \\
\hline \hline N válido & 20 & & & & & & \\
\hline \hline
\end{tabular}

En la tabla 4 se observa que las participantes presentan un nivel moderado de su capacidad para llevar a cabo las iniciativas de negocio y de utilización de los recursos disponibles en cuanto a su capacidad emprendedora $(x=65,85 ; \sigma=5,66)$. Sin embargo, es posible apreciar en la tabla 5 diferencias en las dimensiones de emprendimiento, obteniendo para todas las variables un nivel medio de desarrollo: la primera $(x=6,70$; $\sigma=1,08)$, la segunda $(x=12,10 ; \sigma=1,86)$, la tercera $(x=11,95 ; \sigma=1,54)$, la cuarta $(x=10,55 ; \sigma=1,39)$, la quinta $(\mathrm{x}=12,10 ; \sigma=1,83)$ y la sexta $(\mathrm{x}=12,45$; $\sigma=1,98)$.

Tabla 4. Descriptivos de la variable emprendimiento en las mujeres con discapacidad de la muestra.

\begin{tabular}{lccccccc}
\hline \hline & N & Mínimo & Máximo & Media & Error típico & Desviación estándar & Varianza \\
\hline Nivel de Emprendimiento & 20 & 53 & 76 & 65,85 & 1,27 & 5,66 & 32,03 \\
N válido & 20 & & & & & & \\
\hline \hline
\end{tabular}

Tabla 5. Descriptivos de las dimensiones de la variable emprendimiento en las mujeres con discapacidad de la muestra.

\begin{tabular}{lccccccc}
\hline \hline Dimensiones Emprendimiento & N & Mínimo & Máximo & Media & Error típico & Desviación estándar & Varianza \\
\hline \hline Búsqueda de información & 20 & 5 & 9 & 6,70 & 0,24 & 1,08 & 1,17 \\
Riesgo & 20 & 10 & 16 & 12,10 & 0,42 & 1,86 & 3,46 \\
Perseverancia & 20 & 9 & 14 & 11,95 & 0,34 & 1,54 & 2,37 \\
Autoconcepto & 20 & 9 & 13 & 10,55 & 0,31 & 1,39 & 1,95 \\
Planificación sistemática y & 20 & 8 & 15 & 12,10 & 0,41 & 1,83 & 3,36 \\
seguimiento & 20 & 8 & 15 & 12,45 & 0,44 & 1,98 & 3,95 \\
Cumplimiento & 20 & & & & & & \\
N válido & & & & & & & \\
\hline \hline
\end{tabular}

En la tabla 6 se observa que las mujeres pre- pacidad de considerar de forma positiva sus emosentan un nivel alto del componente afectivo del ciones y sentimientos de satisfacción $(x=21,45$; bienestar subjetivo que tiene relación con la ca- $\sigma=3,96)$.

Tabla 6. Descriptivos de la variable felicidad subjetiva en las mujeres con discapacidad de la muestra.

\begin{tabular}{llllllll}
\hline \hline & N & Mínimo & Máximo & Media & Error típico & Desviación estándar & Varianza \\
\hline \hline Nivel de Felicidad subjetiva & 20 & 14 & 14 & 21,45 & 0,89 & 3,96 & 15,73 \\
N válido & 20 & & & & & & \\
\hline \hline
\end{tabular}


En la tabla 7 se observa que las mujeres presentan un nivel alto del componente cognitivo del bienestar subjetivo que tiene relación con la capacidad de valorar considerar de forma positiva la propia existencia $(x=27,85 ; \sigma=5,79)$.

Tabla 7. Descriptivos de la variable satisfacción vital en las mujeres con discapacidad de la muestra.

\begin{tabular}{lccccccc}
\hline \hline & N & Mínimo & Máximo & Media & Error típico & Desviación estándar & Varianza \\
\hline Nivel de Satisfacción vital & 20 & 11 & 35 & 27,85 & 1,29 & 5,79 & 33,61 \\
N válido & 20 & & & & & & \\
\hline \hline
\end{tabular}

\section{Descripción propuesta de intervención psicosocial}

La propuesta de intervención se formula a partir de los resultados obtenidos en el diagnóstico, identificando en este aquellas variables que se encuentran mayormente debilitadas o en un nivel intermedio que son factibles de mejorar mediante la entrega de herramientas a partir de tres talleres de capacitación a las mujeres con discapacidad intelectual de la muestra explicados a continuación:

Taller 1 Autodeterminación. Busca que las beneficiarias logren un nivel óptimo de su autoconcepto al fortalecer, potenciar o desarrollar su autoestima y aceptación de su condición de discapacidad, considerando que actualmente manifiestan un nivel alto de la variable, pero aún se alejan del puntaje mayor. Es el primer taller porque desarrolla en primera instancia la seguridad en sí mismas de las mujeres, mejorando sus niveles actuales.

Taller 2 Habilidades sociales. Pretende desarrollar en las mujeres de la muestra la capacidad de generar relaciones interpersonales más efectivas y satisfactorias para su desempeño profesional y potenciamiento personal, teniendo en consideración que el nivel global actual es avanzado, aunque no alcanza el puntaje mayor, mientras que por dimensión es moderado en la mayor parte de ellas. Es el segundo taller, ya que primero se requiere que aprendan a autoconocerse para poder establecer interacciones adecuadas con otras personas.

Taller 3 Capacidad emprendedora. Se enfoca en lograr que las beneficiarias adquieran el conocimiento necesario para desenvolverse en futuras proyecciones de negocio propio o de inversión en otros ya existentes, desarrollando sus habilidades microempresarias que actualmente son moderadas y, por ende, factible de ser potenciadas y mejoradas. Es el tercer y último taller, ya que es una habilidad más bien técnica a desarrollar, por lo que se necesita tener solidez en el autoconcepto y en la capacidad de relacionarse con otras personas.

\section{Competencias a desarrollar con la intervención psicosocial}

Mediante la aplicación de los talleres de la intervención, se pretende desarrollar las siguientes competencias en las mujeres con discapacidad intelectual (beneficiarias): 1) Conocer mediante una autoevaluación los niveles de autodeterminación que tiene cada mujer con base en el conocimiento de sus niveles de autoestima y autoconcepto, 2) Gestionar las redes que se encuentran disponibles para facilitar el desarrollo de habilidades sociales y de contacto con personas con la misma condición de discapacidad o sin ella, y 3) Entregar herramientas para que las mujeres sean capaces de manejar su propia microempresa.

\section{Subcompetencias a desarrollar con la intervención psicosocial}

A partir de las competencias desarrolladas en la aplicación de los talleres de la intervención, se pretende desarrollar las siguientes subcompetencias en las mujeres con discapacidad intelectual (beneficiarias): 1) Entregar herramientas para realizar una autoevaluación de los niveles de autoestima en las mujeres, 2) Entregar herramientas para realizar una autoevaluación de los niveles de autoconcepto en las mujeres, 3) Identificar las redes disponibles para realizar gestiones que permitan el desarrollo de la microempresa por parte de las mujeres, 4) Generar una diferenciación entre las redes destinadas a personas con discapacidad versus aquellas no destinadas a personas con discapacidad, 5) Entregar conocimientos en relación a las normativas para iniciar una microempresa, y 7) Capacitar a las beneficiarias en el inicio de actividades de microempresa. 


\section{Metodologías de aprendizaje de la intervención psicosocial}

Las metodologías de aprendizaje que se recomiendan para la intervención están enfocadas y pensadas con base en el conocimiento que existe del nivel de discapacidad que presentan las mujeres de la muestra. De esta manera, tanto las técnicas como las actividades tienen una orientación dinámica y didáctica, como sigue a continuación:

1. Exposiciones temáticas. Su objetivo es entregar conocimientos acerca de la temática tratada para interiorizar las diferentes aristas de la misma. Se utilizará un lenguaje y vocabulario apto para el nivel de discapacidad de las mujeres.

2. Aplicación de instrumentos. Su objetivo es medir algunas de las variables que se quiere reforzar, desarrollar o potenciar en las mujeres a través de instrumentos que utilizan «caritas representativas» como opciones de respuesta.

3. Revisión de documentación de los talleres. Su objetivo es generar un análisis de los datos disponibles que se transformen en un apoyo para el desarrollo de las habilidades.

4. Presentación de vídeos de apoyo. Su objetivo es apoyar directamente los ejercicios y los conocimientos que se entreguen en el taller para que, mediante ejemplos concretos, las mujeres puedan interiorizar de mejor forma lo aprendido.

5. Desarrollo de informativos. Su objetivo es apoyar el desarrollo de las temáticas, quedando como una evidencia tangible para cada beneficiaria que asista al taller.

6. Ejercicios de relajación, respiración e imaginería. Su objetivo es entregar a las mujeres, entre otros elementos, un momento de reflexión durante los talleres y, a su vez, una herramienta automanejable para que logren un mayor autocontrol en relación a la imagen que tienen de sí mismas y hacia su entorno.

7. Desarrollo de casos prácticos. Su objetivo es que las mujeres logren visualizar mediante ejemplos, algunas de las temáticas vistas en los talleres para que interioricen la relevancia de las mismas y coloquen en práctica lo que han podido retener como elementos fundamentales de su aprendizaje.

\section{Planificación de propuesta de intervención psicosocial}

La propuesta de intervención psicosocial está programada para ser realizada en tres módulos con metodología de taller con una duración de 34 horas, divididos en 10 sesiones de tres horas (para Taller 1. Autodeterminación tres horas y para Taller 3. Emprendimiento tres horas) y 4 horas (correspondiente al Taller 2. Habilidades sociales).

\section{Conclusión y discusión}

En la actualidad, persiste la necesidad de elaborar programas destinados a personas con discapacidad, que permitan valorarlos como individuos sociales competentes, subsanando situaciones de discriminación, lo que genera la no inclusión social de las mismas (Cordeu, 2008). Existen variados programas sociales, prelaborales y laborales que atienden necesidades específicas, pero todavía existe una carencia de abordajes integrales que atiendan tanto el desarrollo personal como laboral centrada en la autodeterminación.

En relación a la primera variable, la autodeterminación, se observa que las mujeres con discapacidad intelectual de la muestra, manifiestan un nivel avanzado de su autoestima y de su autoconcepto, lo que indica que, a pesar de tener un grado leve de disfuncionalidad intelectual, son capaces de tener un control de su vida y, además, logran establecer metas de trabajo y toman decisiones en relación a sus responsabilidades (Tamarit, 2005). Cabe destacar, además, que si bien es cierto el resultado indica un desarrollo avanzado de la variable (382,6 puntos) se aleja del valor más alto (506 puntos), por lo que se concluye que es sumamente necesario comenzar potenciando la autodeterminación en las mujeres de la muestra mediante talleres, de tal forma que adquieran la seguridad necesaria en sí mismas que les permita tener su propio desarrollo e inserción laboral en el futuro próximo, mejorando sus niveles actuales.

En cuanto a las habilidades sociales, se puede apreciar que las mujeres de la muestra presentan un nivel global avanzado de su capacidad para interactuar con otros de forma efectiva (91,6 puntos), sin embargo, el puntaje máximo es de 111 puntos, por lo que es necesario reforzar esta variable a través de módulos con 
actividades una vez que el autoconcepto de las mujeres de la muestra se encuentre mayormente desarrollado y potenciado en relación a los niveles actuales, logrando el objetivo planteado por autores como Caballo (1999), quien dice que al tener desarrolladas las habilidades sociales, la persona debe manifestar conductas de respeto hacia los demás y de defensa de los propios derechos. De esta manera, se da un desarrollo moderado de las primeras habilidades sociales, de las habilidades sociales avanzadas y de las habilidades que se utilizan como alternativa a la agresión; mientras que se da un desarrollo con un mayor grado de avance en las habilidades relacionadas con la expresión de sentimientos, la capacidad para enfrentar situaciones estresantes y situaciones en las que se requiere de planificación para llevar a cabo las tareas y responsabilidades expresando así lo que sienten (Combs \& Slaby, 1978).

Ahora bien, respecto de la capacidad emprendedora, se observa que las mujeres de la muestra presentan un nivel global moderado (65,85 puntos) en cuanto a su desarrollo, destacando algunas características que se apreciaron en ellas durante el diagnóstico, planteadas además por Raposo \& do Paço (2011), que son: innovación en la forma de trabajo que tienen, lograr la creación de trabajos personalizados que generan su propio bienestar y satisfacción personal, siendo complementadas con las que se pretenden entregar durante los módulos de intervención, puesto que de esta manera se lograría capacitar a las mujeres para que obtengan un nivel de vida con mayor autonomía a través de sus propios cumplimientos de metas personales (De la Red, Fernández, Marbán \& de la Puente, 2008). Es así que manifiestan un desarrollo medio avanzado frente al cumplimiento de tareas y responsabilidades y un nivel moderado al buscar información, al asumir riesgos, al ser perseverantes, respecto de su autoconcepto y en el seguimiento en sus actividades; por esto es necesario entregar herramientas a las mujeres de la muestra que permitan lograr niveles de desarrollo avanzado respecto de las variables ya mencionadas a través de un módulo de emprendimiento como última herramienta educativa de este estudio, formando profesionales integrales (Verheul, Wennekers, Audretsch \& Thurik, 2001).

En relación al bienestar subjetivo en sus dos dimensiones, felicidad subjetiva y satisfacción vital, se apre- cia, en relación a la primera de ellas, que las mujeres de la muestra manifiestan un nivel alto de desarrollo $(21,45$ puntos), lo que repercute directamente a que son capaces de dar a conocer sus emociones y sentimientos de satisfacción con una disposición de optimismo que les permite considerar su vida como un elemento agradable que les permite ser felices, tal como lo plantean Marinić y Brkljačić (2008). Por otro lado, en relación a la segunda dimensión, satisfacción vital, también existe un nivel alto de desarrollo (27,85 puntos) en las mujeres de la muestra acerca de la valoración que hacen de su propia existencia, hecho que ha sido tratado en varias investigaciones anteriores (para mayor información, ver Diener, 1994; García, 2002; Diener, Suh, Lucas \& Smith, 1999 , García-Viniegras \& González, 2000; Liberalesso, 2002). Aún así, ambos niveles podrían mejorar en mayor medida, logrando en lo posible los valores más altos (para felicidad subjetiva 28 puntos y para satisfacción vital 35 puntos) si se tiene como base un adecuado desarrollo de la autoestima y autoconcepto, la capacidad de relacionarse con otras personas de manera efectiva y teniendo el conocimiento de lo que implica emprender un negocio, puesto que las mujeres lograrían sentirse más felices y satisfechas con su situación sociolaboral. A su vez, es importante mencionar que, al presentar mayores emociones positivas que negativas (Marinić \& Brkljačić, 2008), se pretende mantener, mediante la intervención psicosocial propuesta, estos niveles de sus afectos y aumentarlos dentro de las posibilidades reales que presentan las mujeres de la muestra.

\section{Integración de resultados de diagnóstico y modelo operativo de calidad de vida}

Los tres niveles sociales del modelo operativo de calidad de vida planteado por Schalock y Verdugo (2002/2003) se integran con los resultados obtenidos en el diagnóstico: 1) Primer nivel, microsistema: en este nivel se pueden potenciar las habilidades sociales, la autodeterminación, el bienestar subjetivo y la capacidad emprendedora, donde las tres primeras variables tienen un nivel de desarrollo alto (potenciables en el futuro) y la tercera un nivel de desarrollo moderado, 2) Segundo nivel, mesosistema: en este nivel se pueden potenciar las habilidades sociales y la autodeterminación, donde la primera variable tiene un nivel de desarrollo alto y la segunda un nivel 
de desarrollo moderado, y 3) Tercer nivel, macrosistema: en este nivel se pueden potenciar las habilidades sociales y la capacidad emprendedora, donde la primera variable tiene un nivel de desarrollo alto y la segunda un nivel de desarrollo moderado (ambas potenciables en el futuro).

Las ocho dimensiones planteadas en el modelo operativo de calidad de vida se pueden clasificar según las variables del estudio como sigue:

a. Bienestar subjetivo: 1) Bienestar emocional, 2) Bienestar material y 3) Bienestar físico.

b. Habilidades sociales: 4) Relaciones interpersonales y 5) Desarrollo personal.

c. Autodeterminación: 6) Autodeterminación.

d. Capacidad emprendedora: 7) Inclusión social y 8) Derechos.

Es así que el objetivo principal de la propuesta de intervención psicosocial es entregar herramientas a las mujeres con discapacidad intelectual de la muestra en las variables diagnosticadas con anterioridad que permitan potenciar los niveles ya existentes, desarrollando en mayor medida los elementos que se encuentran más deficientes (nivel moderado).

Es importante considerar en futuras investigaciones la medición de la variable empleabilidad, ya que en las mujeres con discapacidad intelectual de la muestra sólo se midió la capacidad emprendedora, pero no existe conocimiento del nivel de empleabilidad que ellas pueden presentar. Además, es indispensable contar con una base de datos con individuos que se interesen en ser parte de este tipo de estudios a quienes se destinen programas de intervención. Junto con lo anterior, resulta interesante, manteniendo las mismas variables, hacer el diagnóstico con hombres que tengan discapacidad intelectual para comparar resultados en relación al sexo.

\section{Referencias}

ARARTEKO (2003). La integración laboral de las personas con discapacidad en la CAPV. Bilbao: Autor.

Bacon, A., Walker, H., Schwartz, A., O`Hara, D., Calkins, C. \& Wehmeyer, M. (2011). Lessons learned in Scaling Up effective practices: implications for promoting self-determination within developmental disabilities. Exceptionality, 19(1), 46-60.
Boluarte, A., Méndez, J. \& Martell, R. (2006). Programa de entrenamiento en habilidades sociales con retraso mental leve y moderado. Mosaico Científico, 3(1), 34-42.

Caballo, V. (1999). Manual de evaluación y entrenamiento de las habilidades sociales. Barcelona: Siglo XXI.

Colegio de Psicólogos de Chile. (2010). Código de ética. Recuperado de www.colegiopsicologos.cl el 19 de diciembre de 2010.

Combs, T. P. \& Slaby, D. A. (1978). Social skills training with children, en B. Lahey \& A. Kazdin (Eds.): Advances in clinical child psychology. New York: Plenum Press.

Cordeu, C. (2008). Reflexiones en torno a la inclusión sociocomunitaria de personas con discapacidad intelectual: escuchando la voz de sus protagonistas (Tesis para optar al grado de Magíster en Psicología, Universidad de Chile).

Cuadra, H. \& Florenzano, R. (2003). El bienestar subjetivo: hacia una psicología positiva. Revista de $P_{s i-}$ cología de la Universidad de Chile, 12 (1), 83-96.

De la Red, N., Fernández, J., Marbán, J. \& de la Puente, R. (2008). Personas con discapacidad: de los centros ocupacionales a actitudes laborales emprendedoras. Portularia, 8(2), 117-139.

Detrich, R., Keyworth, R. \& States, J. (2008). Advances in evidence-based education: Aroadmap to evidencebased education (vol. 1). Oakland, CA: The Wing Institute.

Diener, E., Emmons, R., Larden, R. \& Griffin, S. (1984). The satisfaction with the escale. Journal of Personality Assessment, 49, 71-75.

Diener, E. (1994). El bienestar subjetivo. Intervención psicosocial. Revista sobre Igualdad y Calidad de Vida, 3(8), 67-113.

Diener, E., Suh, E., Lucas, R. \& Smith, H. (1999). Subjetive well-being. Three Decades of Progress. $P_{S-}$ ychological Bulletin, 125(2), 276-302.

Doll, B., Sands, D. J., Wehmeyer, M. L. \& Palmer, S. (1996). Promoting the development and acquisition of self-determined behavior. En D. J. Sands y M. L. Wehmeyer (Eds.), Self-determination across the life span. Baltimore: P. H. Brookes. 
FONADIS. (2006). Encuesta Casen 2006. Santiago: Gobierno de Chile, Ministerio de Planificación, integra y protege.

García-Viniegras, C. \& González, I. (2000). La categoría bienestar psicológico, su relación con otras categorías sociales. Revista Cubana de Medicina Integral, 16(6), 586-592.

García, M. (2002). El bienestar subjetivo. Escritos de Psicología, 6, 18-39.

Goldstein, A. (1999). Habilidades sociales y autocontrol en la adolescencia. México: Siglo XXI.

González, V. \& Jiménez, A. (2012). Diagnóstico y propuesta de intervención en emprendimiento, autoestima y habilidades sociales en mujeres dirigentes de la Región del Maule. (Tesis para optar al título de Magíster en Psicología Social, mención Gestión y calidad de vida de organizaciones saludables, Universidad de Talca).

Gutiérrez, B. \& Prieto, M. (2002). Manual de evaluación y entrenamiento en habilidades sociales para personas con retraso mental. Consejería de Sanidad y Bienestar Social, Gerencia de Servicios Sociales. Junta de Castilla y León.

Hernández, R., Fernández, C., \& Baptista, P. (1998). Metodología de la investigación. México: McGraw Hill.

Hinojo, F. \& Fernández, F. (2002). Diseño de un programa formativo para la mejora de las habilidades sociales en personas mayores. Pedagogía Social, Revista Interuniversitaria, 9, 309-319.

Junta de Castilla y León. (2004). Proyecto de calidad en los centros de atención a personas con discapacidad intelectual. Consejería de familia e igualdad de oportunidades: Gerencia de Servicios Sociales.

Kuratko, D. F. (2005). The emergence of entrepreneurship education: Development, trends and challenges. Entrepreneurship Theory and Practice, 29(5), 577-598.

La Rosa, J. \& Díaz, R. (1999). Evaluación del autoconcepto: una escala multidimensional. Revista Latinoamericana de Psicología, 23(1), 15-33.

Ledo, C. (2011). Diseño de un proyecto de participación en la comunidad en mujeres con discapacidad intelectual (Trabajo para optar al grado de Terapia ocupacional, Universidad de Coruña).
Lescano, G., Rojas, A., \& Vara, A. (2003). Situación de las habilidades sociales en adolescentes escolares del Perú-2003. Disponible en www.aristidesvara. net/pgnWeb/investigaciones/educacion/HABILIDADES_SOCIALES/habilid_social_02_A.htm [noviembre 11, 2012].

Liberalesso, A. (2002). Bienestar subjetivo en la vida adulta y la vejez: hacia una psicología positiva en América Latina. Revista Latinoamericana de Psicología, 34 (1-2), 55-74.

Lyubomirsky, S. \& Lepper, H. (1999). A measure of subjective happiness: preliminary reliability and construct validation. Social Indicators Research, 46(2), 137-156.

Marinić, M. \& Brkljačić, T. (2008). Love over gold-the correlation of happiness level with some life satisfaction factors between persons with and without physical disability. Journal of Developmental and Physical Disabilities, 20, 527-540.

Montero, D. \& Lagos, J. (2011). Conducta adaptativa y discapacidad intelectual: 50 años de historia y su incipiente desarrollo en la educación en Chile. Estudios Pedagógicos, 37(2), 345-361.

Moyano-Díaz, E. \& Ramos, N. (2007). Bienestar subjetivo: midiendo satisfacción vital, felicidad y salud en población chilena de la Región Maule. Revista Universum, 22(2), 177-193.

Palomo, R. \& Tamarit, J. (2000). Autodeterminación: analizando la elección. Siglo Cero, 189, 21-44.

Peñas, O., Torres, M. \& Gómez, A. (2005). Un modelo de gestión social: la ocupación como factor de inclusión y calidad de vida de población vulnerable. Colombia: Universidad Nacional de Colombia, sede Bogotá.

Raposo, M. \& do Paço, A. (2011). Entrepreneurship education: Relationship between education and entrepreneurial activity. Psicothema, 23(3), 453457.

Schalock, R. L. \& Verdugo, M. A. (2002/2003). Handbook on quality of life for human service practitioners. Washington: American Association on Mental Retardation. Traducción al castellano de M. A. Verdugo y C. Jenaro: Calidad de vida: manual para profesionales de la educación, salud y servicios sociales. Madrid: Alianza. 
Spencer, L. \& Spencer, S. (1993). Competence at work: models por superior performance. New York: John Wiley \& Sons, Inc.

Stancliffe, R. \& Abery, B. (2003). An ecological theory of self-determination: research evidence.In Wehmeyer, M., Abery, B., Mithaug, D. \& Stancliffe, R. (Eds.) (2003). Theory in selfdetermination. Foundations for Educational Practice (pp. 79118). Illinois: Charles C. Thomas Publisher, Ltd.

Tamarit, J. (2001). Propuestas para el fomento de la autodeterminación en personas con autismo y retraso mental. Madrid: CEPRI.

Tamarit, J. (2005). Autismo: modelos educativos para una vida de calidad. Revista de Neurología, 40 (Supl 1), 181-186.

Verdugo, M. A. (n. d.). Autodeterminación y calidad de vida de las personas con discapacidad. Propuestas de actuación. INICO, Universidad de Salamanca.

Verdugo, M., Jordán, B., Vicent, C. \& Martin, R. (2006). Integración laboral y discapacidad. Cornell University ILR School. Gladnet Collection, 235, 1-22.

Verheul, I., Wennekers, S., Audretsch, D., \& Thurik, R. (2001). An eclectic theory of entrepreneurship. Tinbergen Institute Discussion Papers.
Wehmeyer, M. L. (1996). Self-determination as an educational outcome: Why is it important to children, youth and adults with disabilities? In D.J. Sands y M. L. Wehmeyer (Eds.), Self-determination across the life span: Independence and choice for people with disabilities (pp. 15-34). Baltimore, MD: Paul H. Brookes.

Wehmeyer, M. L., Kelchner, K. \& Richards, S. (1996). Essential characteristics of self-determined behavior of individuals with mental retardation. American Journal of Mental Retardation, 100, 632-642. Publicado en español en Siglo Cero, 1996, 168, 17-24.

Wehmeyer, M. L. (2001). Autodeterminación: Una visión de conjunto. En M. A.Verdugo y F. B. Jordán de Urríes (Coords). (1999). Hacia una nueva concepción de la discapacidad (pp. 113-133). Salamanca: Amarú.

Zondek, A., Zepeda, M., González, F. \& Recabarren, E. (2006). Discapacidad en Chile: pasos hacia un modelo integral de funcionamiento humano. Capítulo 1, Discapacidad, apoyo social y calidad de vida. Santiago: FONADIS. 